\title{
AN UPPER BOUND FOR THE ZEROS OF THE CYLINDER FUNCTION $C_{v}(x)$
}

\author{
ÁRPÁD ELBERT AND ANDREA LAFORGIA
}

Abstract. For large values of $v(v>0)$ the $k$-th positive zero of the cylinder function $C_{V}(x)=J_{V}(x) \cos \alpha-Y_{V}(x) \sin \alpha, 0 \leqslant \alpha<\pi$, has the asymptotic expansion

$$
j_{v \kappa}=v+\gamma_{\kappa} v^{1 / 3}+\frac{3}{10} \gamma_{\kappa}^{2} v^{-1 / 3}+\mathcal{O}\left(v^{-1}\right)
$$

where $\kappa=k-\alpha / \pi, \gamma_{\kappa}=-a_{\kappa} 2^{-1 / 3}$ and $a_{\kappa}$ is the $k$-th negative zero of the function $A i(x) \cos \alpha+B i(x) \sin \alpha$ and $A i(x), B i(x)$ denote the Airy functions of the first and the second kind, respectively [1]. We prove that the sum of the first three terms of the asymptotic expansion gives an upper bound for $j_{v \kappa}$, provided $\gamma_{\kappa} \geqslant \sqrt[3]{35 / 4}=2.0606427 \ldots$ or $\kappa \geqslant \kappa_{0}=$ $1.13019788 \cdots=2-\alpha_{0} / \pi$ where $\alpha_{0}$ is determined by the equation $\cos \alpha_{0} A i(-\sqrt[3]{35 / 4})+$ $\sin \alpha_{0} B i(-\sqrt[3]{35 / 4})=0$. This result covers the cases $j_{v 2}, j_{v 3}, \ldots$ and $y_{v 2}, y_{v 3}, \ldots$, for all $v>0$. The main tool used is the well-known Watson formula for $d j_{v \kappa} / d v$.

Mathematics subject classification (1991): 41A60, 33C45.

Key words and phrases: Cylinder functions, zeros, asymptotic expansions.

\section{REFERENCES}

[1] M. Abramowitz And I. A. Stegun, eds., Handbook of mathematical functions, Dover Publications, Inc., New York, 10th ed., 1972.

[2] Á. Elbert AND A. LAFORGiA, A lower bound for the zeros of the Bessel functions, World. Sci. Ser. Appl. Anal. 3 (1994), 179-185.

[3] - Asymptotic expansions for zeros of Bessel functions and of their derivatives for large order, Rendiconti del Seminario Matematico e Fisico dell' Università di Modena, to appear.

[4] _ On the square of the zeros of Bessel functions, SIAM J. Math. Anal. 15 (1984), 206-212.

[5] T. LANG AND R. WONG, "Best possible" upper bounds for the first two positive zeros of the Bessel function $J_{v}(x)$ : the infinite case, J. Comput. Appl. Math. 71 (1996), 311-329.

[6] L. LORCH AND R. UBERTI, "Best possible" upper bounds for the first positive zeros of Bessel functions — the finite part, J. Comput. Appl. Math. 75 (1996), 249-258.

[7] G. N. WATSON, A treatise on the theory of Bessel functions, 2nd. ed., Cambridge University Press, London and New York, 1944. 